\title{
Robot-assisted Ultrasound-guided Tracking of Anatomical Structures for the Application of Focused Ultrasound
}

Innovation Center Computer Assisted Surgery, Leipzig, Germany, e-mail: michael.unger@medizin.uni-leipzig.de Johann Berger, Innovation Center Computer Assisted Surgery, Leipzig, Germany

Bjoern Gerold: Theraclion SA, Malakoff, France

Andreas Melzer, Innovation Center Computer Assisted Surgery, Leipzig, Germany

\section{Introduction}

High intensity focused ultrasound is used as a surgical tool to treat completely non-invasively several diseases. The induced thermal and mechanical effects enable the treatment of a variety of pathologies, like uterine fibroids, prostate cancer, thyroid nodules, and varicose veins. Precise targeting is key for improving the treatment outcome A method for an automated, robot-assisted tracking system was developed and evaluated. A wireless ultrasound scanner was used to acquire images of the target, in this case, a blood vessel.

\section{Methods}

A Clarius L7 (Clarius Inc., Canada) wireless ultrasound scanner was used to acquire images of the great saphenous vein (GSV). The active contour approach by Chan and Vese was used to segment and track the vessel. The user needs to specify the vein in the first image. Subsequently, the vesel is tracked automatically. The segmentation performance was assessed by comparing the results of the algorithm to the results of manual segmentation. Furthermore, the ultrasound scanner was attached to a KUKA LBR Med robotic arm (KUKA AG, Germany). The scanner was positioned on an Agar phantom containing a $10 \mathrm{~mm}$ duct filled with ethanol representing an artificial vessel. While moving the ultrasound scanner along the direction of the vessel, the lateral position was corrected using the segmentation results. The performance was assessed by manually segmenting the 50 images acquired during the tracking. The tracking error is defined as the remaining distance of the lesion to the images' centre line.

\section{Results}

The segmentation error of the GSV data set was $0.47 \pm 0.39 \mathrm{~mm}$ (mean \pm std) and $0.48 \pm 0.39 \mathrm{~mm}$ for the phantom. The mean tracking error was $0.27 \pm 0.18 \mathrm{~mm}$ and the mean segmentation time was $0.42 \mathrm{~s}$.

\section{Conclusion}

Active contours were used to track the GSV in ultrasound images. The position of the segmented vein can be used to correct the position of an ultrasound-guided HIFU treatment unit. 


\section{Robotic Assistance System for Cone Beam Computed Tomography- guided Needle Placement}

Andreas Rothfuss, BEC GmbH, Pfullingen, Germany, a.rothfuss@b-e-c.de

Philipp Lautenschläger, Fraunhofer IPA, Mannheim, Germany, philipp.lautenschlaeger@ipa.fraunhofer.de

Simon Störk, BEC GmbH, Pfullingen, Germany, s.stoerk@b-e-c.de

Dr.-Ing. Markus Kühne, Fraunhofer IPA, Mannheim, Germany, markus.kuehne@ipa.fraunhofer.de

Matthias Buck, BEC GmbH, Pfullingen, Germany, m.buck@b-e-c.de

Prof. Dr.-Ing. Jan Stallkamp, Fraunhofer IPA, Mannheim, Germany, jan.stallkamp@ipa.fraunhofer.de

\section{Introduction}

Due to an increase in applications and indications, minimally invasive percutaneous interventions are being performed more frequently in clinical routine. The guidoo system, currently under development at BEC GmbH (Pfullingen, Germany) and Fraunhofer IPA (Mannheim, Germany), aims to provide an easy to use and fast approach to these interventions.

\section{Methods}

A lightweight robot (LBR med, KUKA AG, Germany) equipped with a robotic end-effector specifically designed for the task was used in conjunction with a CBCT interventional suite (Artis zeego, Siemens Healthineers, Germany; Magnus OR-Table, Maquet GmbH, Germany) and dedicated navigation software. A group of 20 medical students was divided into two groups to asses the precision, procedural duration and subjective confidence of the robot-assisted procedure in comparison to the procedure guided by the Siemens NeedleGuidance ${ }^{\text {TM}}-$ System (NG) in a phantom-based setup.

\section{Results}

Comparing the results for all 349 sucessfully placed needles, the deviation of the needle tip does not differ significantly between both systems (NG: 2,62 $\pm 1,34 \mathrm{~mm}, 0,60-9,43 \mathrm{~mm}$, guidoo: 2,98 mm $\pm 1,38 \mathrm{~mm}, 0,78-7,96 \mathrm{~mm}$ ). The overall procedural time does not differ significantly as well (NG: $602 \pm 226 \mathrm{~s}, 328-1734 \mathrm{~s}$, guidoo: $548 \pm 158 \mathrm{~s}, 341-1232 \mathrm{~s}$ ), while the time required to place a single needle (NG: $174 \pm 144 \mathrm{~s}, 12-957$ s, guidoo: $28 \pm 39 \mathrm{~s}, 3-262 \mathrm{~s}$ ) is significantly lower for the robot-assisted procedure. Even though the confidence is high for both systems, the measured confidence for the robotic system is significantly higher (NG: 79,9 $\pm 13,4 \%, 37,0-100,0 \%$, guidoo: 84,3 $\pm 12,1 \%, 44,4-100,0$ $\%)$.

\section{Conclusion}

The precision and overall procedural times are similar for both setups, while the robot-assisted system requires less time to place a single needle. This points to a potential advantage for the robotic system in percutaneous interventions requiring multiple needle placement like Irreversible Electroporation and LDR Brachytherapy. In addition to this, the higher confidence with the robotic system also points to a potential advantage for less experienced interventionalist using the system. 


\section{A Collaborative and Integrated Robotic System for the Application in Im- age-Guided Interventions}

Johann Berger, Innovation Center Computer Assisted Surgery (ICCAS), University Leipzig, Leipzig, Germany, johann.berger@medizin.uni-leipzig.de

Johannes Keller, Innovation Center Computer Assisted Surgery (ICCAS), University Leipzig, Leipzig, Germany, johannes.keller@medizin.uni-leipzig.de

Michael Unger, Innovation Center Computer Assisted Surgery (ICCAS), University Leipzig, Leipzig, Germany, michael.unger@medizin.uni-leipzig.de

Lisa Landgraf, Innovation Center Computer Assisted Surgery (ICCAS), University Leipzig, Leipzig, Germany, lisa.landgraf@medizin.uni-leipzig.de

Andreas Melzer, Innovation Center Computer Assisted Surgery (ICCAS), University Leipzig, Leipzig, Germany, andreas.melzer@medizin.uni-leipzig.de

\section{Introduction}

Accurate tool positioning is a key requirement to perform image-guided interventions like focused ultrasound ablations. Collaborative robots provide precise tools to perform sophisticated procedures. However, currently available systems are often limited to single use-cases and high costs restrict their deployment in the clinic. The IEEE 11073 SDC standard for a service-oriented device connectivity that was developed in the OR.NET project, allows for a vendorindependent communication between devices. It may, therefore, help to integrate robotic systems into the operating room. This work presents an integrated robotic dual-arm system (DAS) to overcome named problems.

\section{Methods}

The system consists of two KUKA LBR iiwa 7 R800 robotic arms (KUKA AG, Germany), mounted on two mobile platforms. It is intented to be usable in different use-cases, by modeling both robots as a single medical device system as service provider inside the SDC standard. It can, therefore, be accessed by any standard conform user application to perform different tasks for specific medical scenarios. The robots were co-registered using an NDI Polaris Vicra tracking camera (Northern Digital Inc., Canada). Including an augmented-reality based planning visualization the system's usability was tested in two use-cases for ultrasound image guided biopsies and the application of ultrasound guided focused ultrasound (FUS). The system's accuracy was evaluated by measuring the co-registration for three different landmark-registration approaches. By attaching a reference tool to the robots, the interdependent positioning error from one robot to another was recorded for 10 different positions with the NDI camera.

\section{Results}

Including collaborative functionality, both robots can be steered with hand-guidance and touch-gestures and an automated movement to position the interventional tools is included as well. The worst registration method produced a maximum error of $4.17 \mathrm{~mm}$. The best registration was measured with an error of $2.58 \mathrm{~mm}$.

\section{Conclusion}

The feasibility of using an integrated dual-arm robotic system for multiple use-cases was shown. To provide a proof of concept, two different image-guided intervention scenarios were presented. The recorded error of $2.58 \mathrm{~mm}$ is sufficient for FUS treatments of targets with a $3 \mathrm{~mm}$ size or more. This work is a preliminary study and, therefore, does not include user behavior. A study with different users is underway. 


\section{Telemanipulation of an Articulated Robotic Arm using a Commercial Virtual Reality Controller}

Max B. Schäfer, Institute of Medical Device Technology, University of Stuttgart, max.schaefer@imt.uni-stuttgart.de Kent W. Stewart, Institute of Medical Device Technology, University of Stuttgart, kent.stewart@imt.uni-stuttgart.de Nico Lösch, Institute of Medical Device Technology, University of Stuttgart, st115472@stud.uni-stuttgart.de Peter P. Pott, Institute of Medical Device Technology, University of Stuttgart, peter.pott@imt.uni-stuttgart.de

\section{Introduction}

Recently, a strong emergence in teleoperated minimally invasive surgery is observed due to the potential benefits in surgeon's performance and patient outcomes. However, access to such systems is limited due to high acquisition and operating costs. Current systems commonly use universal linkage-based input devices, while only few application-oriented and specialized device designs were found. A versatile Virtual-Reality-controller (VR) is presented as an alternative input device. The real-time capabilities of the setup, replicating a system for robot-assisted teleoperated surgery, are investigated to assess the suitability of the VR-controller as the input device.

\section{Methods}

A 7-DOF articulated robotic arm (Panda, Franka-Emika GmbH, Munich, Germany) and an HTC Vive VR-controller (High-Tech-Computer-Corporation, Taoyuan-City, Taiwan) were used in a master-slave telemanipulation setup. Impedance control was used for endeffector positioning, enabling collaborative use and interaction with objects of varying stiffness. Overall system-latency was assessed using visual markers on the controller and robotic endeffector, determining the delay between motion of both markers in camera images (sampling rate $240 \mathrm{~Hz}$ ). In addition, the control algorithm delay was assessed with a step and raised cosine input function and the robot's position feedback.

\section{Results}

Translation and rotation of the VR-controller were successfully mapped to the endeffector pose. By image-based assessment, the overall system showed a mean latency of $81.7 \pm 27.7 \mathrm{~ms}$. Further, the control algorithm showed a mean response time of $12.0 \pm 0.0 \mathrm{~ms}$ for the step function and $70.2 \pm 1.3 \mathrm{~ms}$ for the raised cosine.

\section{Conclusion}

A telemanipulation system for research around robot-assisted surgery was set up using low-cost and commercially available equipment. The VR-controller is a promising alternative to current input devices for medical telemanipulation systems. However, drawbacks such as the considerable latency and the input device ergonomics need to be further addressed. 


\section{Multipolar Stimulation Probe for Robotic Cochlear Implantation}

Celine Wegner, inomed Medizintechnik GmbH, Emmendingen, Germany, c.wegner@inomed.com Maria Rombach, inomed Medizintechnik GmbH, Emmendingen, Germany Juan Anso, ARTORG Center, University of Bern

Andreas Holzner, CAScination AG, Bern, Switzerland, andreas.holzner@cascination.com

Marco Matulic, CAScination AG, Bern, Switzerland, marco.matulic@ cascination.com

Stefan Weber, CAScination AG, Bern, Switzerland, stefan.weber@cascination.com

Cilgia Dür, Inselspital Bern, Switzerland

Frédéric Venail, CHU Montpellier, France

Thilo Krüger, inomed Medizintechnik GmbH, Emmendingen, Germany, t.krueger@inomed.com

\section{Introduction}

In Robotic Cochlear implantation a $1.8 \mathrm{~mm}$ tunnel to the tympanic cavity in $0.4 \mathrm{~mm}$ distance to the facial nerve is robotically drilled. As the surgeon cannot directly monitor the structural preservation of the facial nerve, an alternative monitoring system is required. For this electromyographic facial nerve monitoring was previously proposed and clinically introduced. The system consists of multipolar stimulation and electrophysiologic recording, where the stimulation probe is placed in the tunnel and resulting muscle contractions are measured. The probe consists of an anode tip for monopolar stimulation at the distal end and three cathode electrode rings for bipolar stimulation. The multipolar approach enables the combination of the desired characteristics of the monopolar stimulation for high sensitivity with the high specificity provided by bipolar stimulation. In this project, the existing research design was transferred to a commercially available medical product and was financially supported through a Eurostars research fund (E!11597).

\section{Methods}

The previously established design served as the design input for the development of the probe in compliance with regulatory requirements (Directive 93/42/EEC). A neurostimulator and amplifier (ISIS Neurostimulator and ISIS Headbox, inomed Medizintechnik GmbH) were integrated into the robotic system (HEARO, CAScination AG). The capability of the approach to allow a surgeon to discriminate safe vs. unsafe trajectories by interpretation of the nerve responses was investigated in a single-blinded prospective in-vivo animal study.

\section{Results}

The study findings demonstrate that it was possible for the surgeons to correctly identify safe drilling trajectories from otherwise unsafe drilling trajectories, in which the drilling was aborted. The translational work from research to a medical device was successfully completed and the CE-mark of the overall robotic system was achieved.

\section{Conclusion}

The system is currently under clinical investigation (NCT04102215, ClinicalTrials.gov). Further investigation on reducing the probe diameter allowing for smaller drill tunnels is ongoing. 